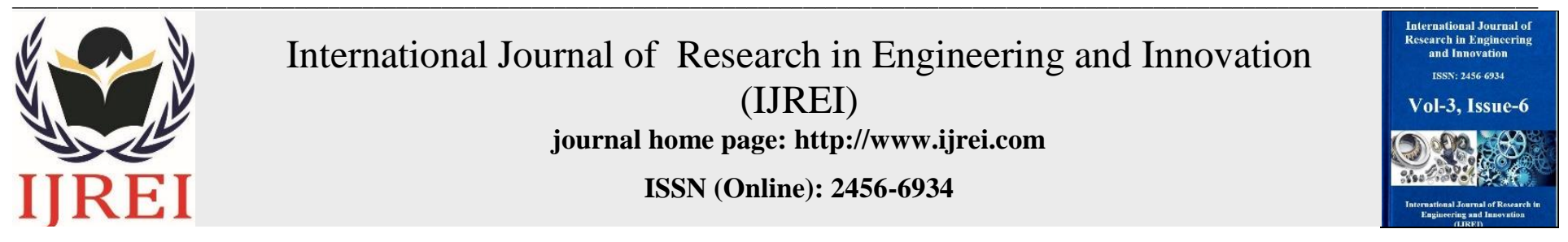

\title{
A non-LTE plasma nuclear physics, kinetics and line shape set
}

\author{
Hind Kh. Abbas
}

University of Karbala, Department of physics, Karbala, Iraq.

\begin{abstract}
ALICE is a plasma nuclear behavior, nuclear kinetics and line shape set: it computes the energy stages and irritated units for changes in an assumed ion which are then secondhand to do a nuclear kinetics control to discovery the inhabitants of those heights, and secondhand to crop the opaqueness of the physical. The energy arena shaped is nourished spinal self-consistently and spectrally determined into the nuclear kinetics to compute the tricking of the energy in a assumed geometry, and the whole tricky is resolved period powerlessly for also a solitary physical or a combination. Completely three chunks of a nuclear physics, nuclear kinetics and line shape encryption, ALICE, are defined. Samples of the code existence secondhand to perfect opaqueness of plasmas are deliberated and stimulating topographies of the encryption which shape on the current quantity of replicas are exposed through.

Keywords: Kinetics, Physics Nuclear, ALICE

(c) 2019 ijrei.com. All rights reserved
\end{abstract}

\section{Introduction}

ALICE was intended to perfect trials on the ORION petite beat laser scheme at AWE and comprises numerous alterations to explanation for belongings on the vigor heights and line shapes in thick plasmas. New outcomes in the governments retrieved in those trials will be likened to ALICE in the study. Numerous full nuclear behavior encryptions for scheming the energy heights in ions and the charges among them have been inscribed, for instance the encryptions [1, 4], FAC; and there are also big, fewer full codes consuming logical estimates, for instance GALAXY, and additional incomplete cunnings did in LTE when the filled praise of charges are not obligatory, for instance DAVROS [7-8]. There are several nuclear kinetics replicas in custom, frequently sketch on a nuclear physics code or by means of the effort from such a code, for instance. Finally, numerous line shape replicas be, both combined hooked on code like NUCLEAR or as separate codes of variable heights of complexity $[2,6]$.

\section{Methodology}

Collisional cross units Measure In the control of collisional crossunits, the chief tricky is the control of the circular integral, and in specific attainment meeting of the amount ended bony impetuses of the allowed electrons in the double magnate change circumstance. The minimization of the amount of circular integrals which necessity be intended has remained the topic of substantial training connecting the cut of the amount of bony impetuses as initial as likely and the control of the cross-unit at the minimum likely amount of dynamisms to minimize the sum of like additions reliable with a adequately precise consequence [3]. ALICE deceits among the two methods of also scheming numerous opinions of the cross-unit and then execution a meansquares appropriate to person's opinions though in approximately suitcases annoying to uphold nearly bodily bounds, and scheming a humble cross-unit and then smearing scaling's to it in instruction to copy behavior realized in additional cunnings. Model covenant is mention in Figure 1 whole arrangement interaction is involved and ever after the whole dispersal of the stages is 13:7 Ryd, the energy variation of alice are all $<0: 3 \%$ genealogical to that dispersal.

We wish to evade scheming slanted upsurge crash fortes, chiefly those for the dipole changes and chiefly at dynamisms meaningfully overhead verge. This is since at person's dynamisms, as well as an upsurge in the effort of the arithmetical control owing to the extremely oscillatory countryside of the purposes, the amount of incomplete surfs which necessity be summed develops actual big. 


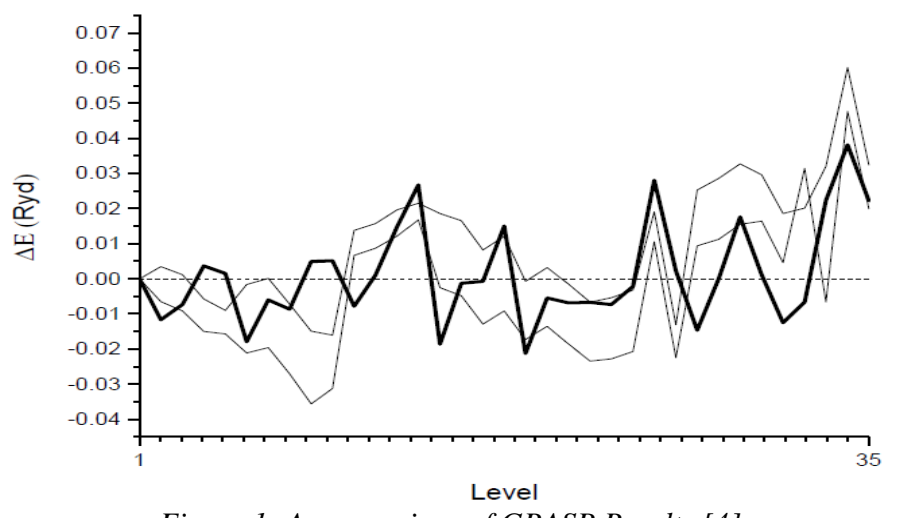

Figure 1: A comparison of GRASP Results [4]

\section{Results and Discussion}

\subsection{Relativistic formulae}

Owing to the usage of a careful great energy boundary by the constants $\mathrm{c} 1$ and $\mathrm{c} 2$, the formulations exposed overhead spread to the relativistic circumstance. For the double pole allowable changes, counting accuracy belongings on the allowed electron. Approximately instances of the cross-sections intended by means of this technique are shown in Fig. 2:

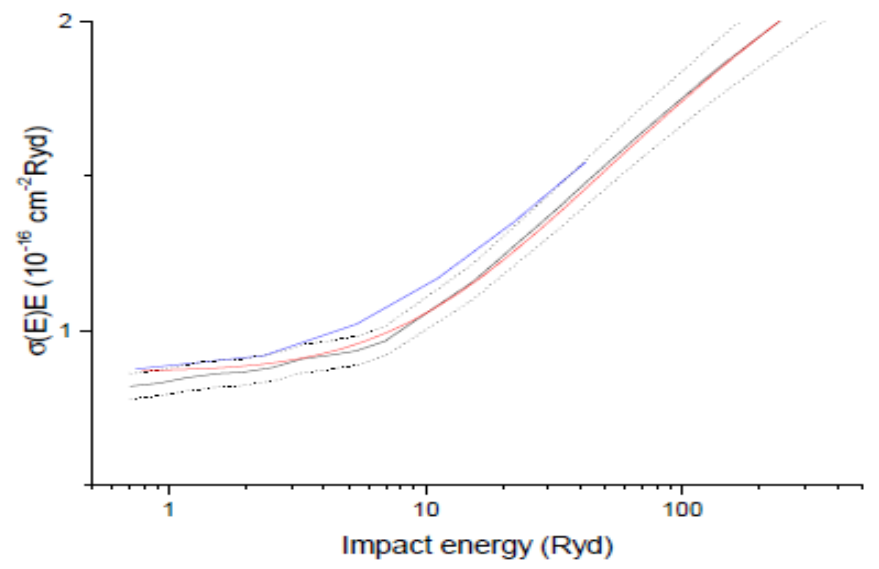

(a)

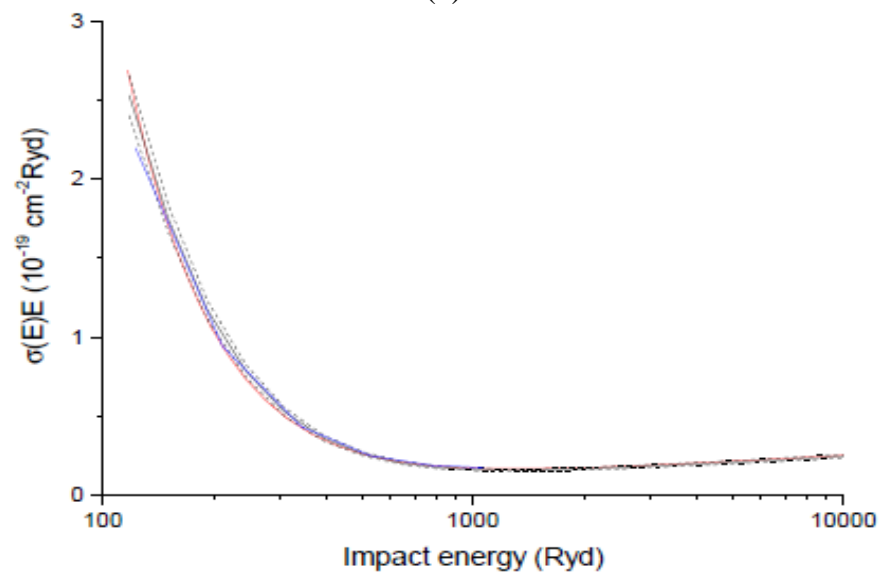

(b)

Figure 2: Results of Relativistic formulae

\subsection{Auto ionization rates}

These are envisioned in ALICE by separate the four dynamic electrons procurement a dual quantity over small paternity constants, and an amount of $6 \mathrm{j}$ and $9 \mathrm{j}$-codes. These environment features consume remained authenticated in contradiction of those from FAC, and, though the standards vary somewhat owing to the dangerous compassion to the circular matrix features, an general lined association among the standards can be understood as is exposed in Fig. 3.

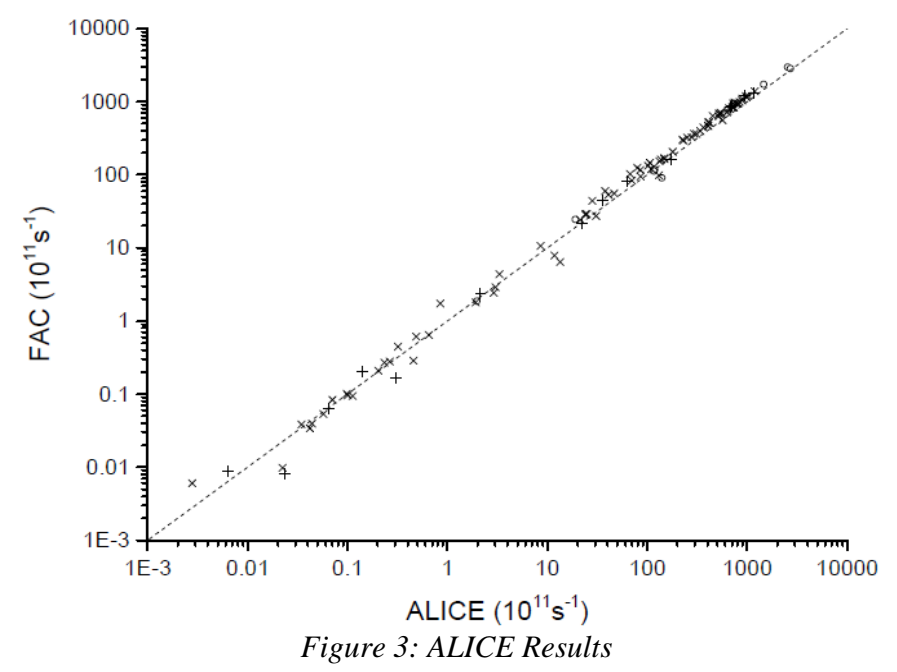

\section{Conclusion}

In this study we have obtainable the bodily replicas castoff in the non-LTE plasma nuclear behavior, kinetics and line shape set ALICE and established the essential for those replicas in circumstances of attention by contrasts of ALICE's production together beside that of additional cyphers and in contradiction of new facts. We have exposed a competence to perfect in LTE at little thickness with the estimated presence of Rydberg conditions, an exposed L-shell competence at hard thickness, the demonstrating of period reliant on non-LTE glitches in resources where the energy conveyance in non-logical line shapes is significant and the essential to comprise advanced instruction multi-pole quasistatic ion expansion to replicate experimentally experiential ranges after hard thickness plasmas.

\section{References}

[1] Ahmad M. Karim, Mehmet S. Güzel, Mehmet R. Tolun, Hilal Kaya, and Fatih V. Çelebi, "A New Generalized Deep Learning Framework Combining Sparse Autoencoder and Taguchi Method for Novel Data Classification and Processing," Mathematical Problems in Engineering, vol. 2018, Article ID 3145947, 13 pages, 2018.

[2] Ahmad M. Karim, Mehmet S. Güzel, Mehmet R. Tolun, Hilal Kaya, Fatih V. Çelebi, A new framework using deep auto-encoder and energy spectral density for medical waveform data classification and processing, Biocybernetics and Biomedical Engineering, Vol 39 (1), 148-159, 2019.

[3] A. M. Karim, F. V. Çelebi, and A. S. Mohammed, "Software Development for Blood Disease Expert System," Lecture Notes on Empirical Software Engineering, vol. 4, no. 3, pp. 179-183, 2016. 
[4] E.G. Hill, G. Pérez-Callejo, S. J. Rose, ALICE: A non-LTE plasma atomic physics, kinetics and lineshape package, High Energy Density Physics, Volume 26, 56-67, 2018.

[5] Hind Khudheyer, Hisham Jashami, A new method combining discrete wavelet transform and neural network for high energy physics problem, International journal of research in engineering and innovation (IJREI), vol 3 , issue 2, 79-81, 2019.
[6] Hind Khudheyer, Hisham Jashami, Support vector machine based on power of signal for solving high-energy physics problem, International journal of research in engineering and innovation (IJREI), vol 3, issue 1, 57-60, 2019.

[7] A. M. Karim, F. V. Celebi, and A. S. Mohammed, "Software Development for Blood Disease Expert System," Lecture Notes on Empirical Software Engineering, vol. 4, no. 3, pp. 179-183, 2016.

[8] A. M. Karim, Ö. Karal, and F. V Çelebi, “A New Automatic Epilepsy Serious Detection Method by Using Deep Learning Based on Discrete Wavelet Transform," no. 4, 15-18, 2018.

Cite this article as: Hind Kh. Abbas, A non-LTE plasma nuclear physics, kinetics and line shape set, International Journal of Research in Engineering and Innovation Vol-3, Issue-6 (2019), 377-379. http://doi.org/10.36037/IJREI.2019.3604. 